\title{
A machine learning management model for QoE enhancement in next-generation wireless ecosystems
}

\author{
Eva Ibarrola \\ University of the Basque Country \\ Mark Davis \\ Technological University Dublin, mark.davis@tudublin.ie \\ Camille Voisin \\ OptiWi-fi, Ireland
}

See next page for additional authors

Follow this and additional works at: https://arrow.tudublin.ie/engscheleart

Part of the Electrical and Computer Engineering Commons

\section{Recommended Citation}

E. Ibarrola, M. Davis, C. Voisin, C. Close and L. Cristobo, "A Machine Learning Management Model for QoE Enhancement in Next-Generation Wireless Ecosystems," 2018 ITU Kaleidoscope: Machine Learning for a 5G Future (ITU K), 2018, pp. 1-8, doi: 10.23919/ITU-WT.2018.8598032.

This Conference Paper is brought to you for free and open access by the School of Electrical and Electronic Engineering at ARROW@TU Dublin. It has been accepted for inclusion in Conference papers by an authorized administrator of ARROW@TU Dublin. For more information, please contact arrow.admin@tudublin.ie, aisling.coyne@tudublin.ie,gerard.connolly@tudublin.ie.

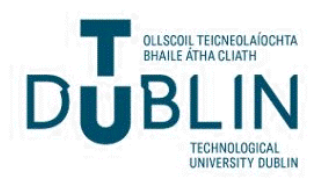




\section{Authors}

Eva Ibarrola, Mark Davis, Camille Voisin, Ciara Close, and Leire Cristobo 


\title{
A MACHINE LEARNING MANAGEMENT MODEL FOR QoE ENHANCEMENT IN NEXT-GENERATION WIRELESS ECOSYSTEMS
}

\author{
Eva Ibarrola ${ }^{1}$, Mark Davis ${ }^{2}$, Camille Voisin $^{3}$, Ciara Close $^{3}$, Leire Cristobo $^{1}$ \\ ${ }^{1}$ University of the Basque Country (UPV/EHU), Spain \\ ${ }^{2}$ Dublin Institute of Technology (DIT), Ireland \\ ${ }^{3}$ OptiWi-fi, Ireland
}

\begin{abstract}
Next-generation wireless ecosystems are expected to comprise heterogeneous technologies and diverse deployment scenarios. Ensuring a good quality of service $(Q o S)$ will be one of the major challenges of nextgeneration wireless systems on account of a variety of factors that are beyond the control of network and service providers. In this context, ITU-T is working on updating the various Recommendations related to QoS and users' quality of experience (QoE). Considering the ITU-T QoS framework, we propose a methodology to develop a global QoS management model for next-generation wireless ecosystems taking advantage of big data and machine learning. The results from a case study conducted to validate the model in real-world Wi-Fi deployment scenarios are also presented.
\end{abstract}

Keywords - Big data, machine learning, QoBiz, QoE, QoS, Wi-Fi

\section{INTRODUCTION}

The evolution of Internet users' behavior in recent years, along with the increasing variety of free applications and services, has led to Internet access becoming something indispensable for our daily life. As a result, users are turning out to be more and more demanding in terms of Internet coverage, accessibility and mobility, causing Internet service providers (ISPs) to consider alternative business models to fulfill these needs. For this reason, some of the technologies that were originally considered for providing local access to the Internet have now emerged as ubiquitous access technologies, leading to complex scenarios where fulfilling the required quality of service (QoS) will become a real challenge.

The Wi-Fi technology, defined in the IEEE 802.11 standard, is a good example of this. While originally designed to be a wireless local area network (WLAN) technology, today's large deployment of Wi-Fi networks has encouraged ISPs to consider new business models turning this technology into a "ubiquitous access technology for mobile users" [1] In this way, providers can offer complementary broadband access ensuring the coverage, mobility and accessibility demanded by their users. Consequently, many scientific and industrial researchers [2] envisage 5G as having an agnostic radio access network (RAN) comprising multiple wireless technologies.

Therefore, even though there is still no clear consensus about what the next-generation wireless (NGW) era will embrace, there seems to be a general agreement that $5 \mathrm{G}$ will comprise heterogeneous networks (HetNet) cooperating to maintain a user's QoE. Furthermore, the adoption of new business models, quality of business (QoBiz), to integrate all the capabilities that next-generation wireless systems may offer will be crucial. Nonetheless, ensuring the required quality of experience in these complex scenarios will become a major issue.

The ITU's standardization expert group for future networks (SG-13) has been working towards the definition of $5 \mathrm{G}$ systems and the development of new Recommendations related to QoS in the NGW environment $[3,4]$. In addition, being aware of the great challenge of managing next-generation wireless networks, new groups, like the "Focus Group on Machine Learning for Future Networks including 5G" [5], have been established. ITU-T SG-12 has also focused on updating and defining new Recommendations related to QoS and QoE for adapting to the new NGW scenario [6, 7]. Nevertheless, there is still a need for methodologies that will take advantage of new techniques and mechanisms, such as machine learning (ML) algorithms, for the deployment of QoS management models as defined in the standardized QoS frameworks.

In this paper, a methodology for the implementation of a global QoS management model in NGW ecosystems is proposed. The model takes into account the ITU QoS framework [8] and the methodology aims to include all the aspects that the $5 \mathrm{G}$ era will require. Enhancing the quality of experience and the satisfaction of the users through new business models to fulfill their requirements are the main target of the methodology. The identification of the optimal key performance indicators (KPIs) and key quality indicators (KQIs) are essential to achieve this goal. 
The proposed methodology represents a major challenge because of the unpredictable nature of the scenarios considered, with networks sharing the spectrum (even working in unlicensed bands) and a response totally dependent on the behavior of users and many other contextual and non-contextual agents not controlled by the providers. Addressing this challenge through ML constitutes the novelty of this proposal.

The remainder of the paper is organized as follows: Section 2 summarizes related work on QoS management approaches in wireless scenarios considering the use of ML-based models, and also standardization-related works are reviewed. Section 3 describes the QoS management model that is adopted and defines the QoS-QoE-QoBiz relations to be considered. In Section 4, the proposed methodology to implement the model in NGW scenarios through ML mechanisms is described. Section 5 illustrates the experiment carried out to validate the proposed methodology and, finally, Section 6 contains some conclusions and final remarks.

\section{BACKGROUND}

Many studies and standardization efforts related to QoS and QoE have been conducted in recent years. Most of these show that the assessment of QoS has moved away from network performance (NP) in favor of QoE, related to the subjective perception of end users. However, as stated in the Recommendation ITU-T G.1000 on the QoS framework [8], the great challenge to success when deploying a QoS management model is to embrace all the different QoS-related aspects (e.g. NP, QoS, QoE, QoBiz) and, more importantly, quantifying the relationships between them. This may become a difficult task when dealing with next-generation wireless ecosystems, where many unpredictable instances may have an influence on the user's experience.

In view of this, some recent studies suggest using big data analysis and ML algorithms for modeling the QoE-QoS relationship. ML techniques may be useful to infer rules from big data analysis and identify the KPIs/KQIs that will lead to automatically estimating the quality as perceived by users based on the QoE influence factors. Selecting the suitable learning algorithm may be critical to obtaining reliable results.

\subsection{QoE models and machine learning}

There is limited research on the modeling of QoE with ML for next-generation mobile scenarios. Nevertheless, there are some recent studies focused on analyzing and using the data captured from the network and the users' surveys to model and to enhance the QoE [9-11].

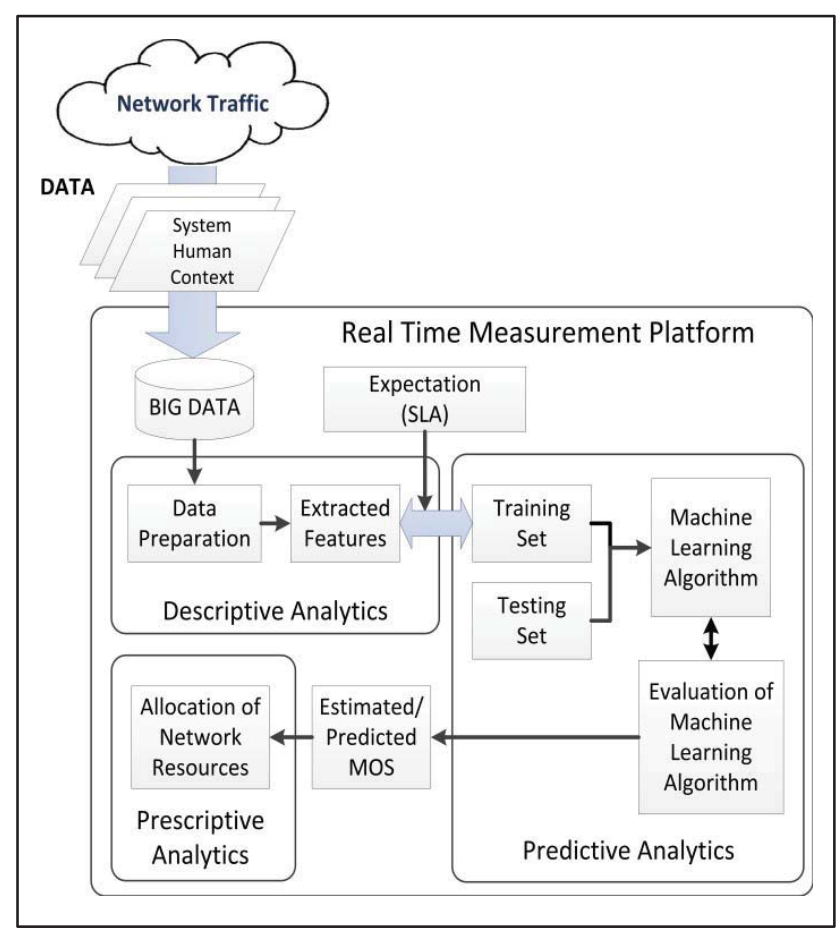

Figure 1. Framework for modeling the perceived QoE using a big-data analytics approach [9]

The framework presented in [9] (Figure 1) is a step ahead of the other works. In the proposed framework, "the process of estimating or predicting the perceived QoE based on the datasets obtained or gathered from the mobile network to enable the mobile network operators effectively to manage the network performance and provide the users a satisfactory mobile Internet QoE" is described. The state-of-the-art included in this work covers a large set of experiments in different scenarios and for different applications and services in relation to modeling QoE with ML algorithms. Their study about the dimensions of the QoE influence factors reviews a great number of scientific proposals. After the analysis, the authors concluded that three QoE dimensions (human, system, and context) should be considered when modeling QoE (Figure 2).

Aroussi and Mellouk presented in [10] an interesting survey about ML-based QoE-QoS correlation models. They suggest that supervised or semi-supervised learning models provide a better fit for the QoE-QoS correlation modeling than non-supervised ML.

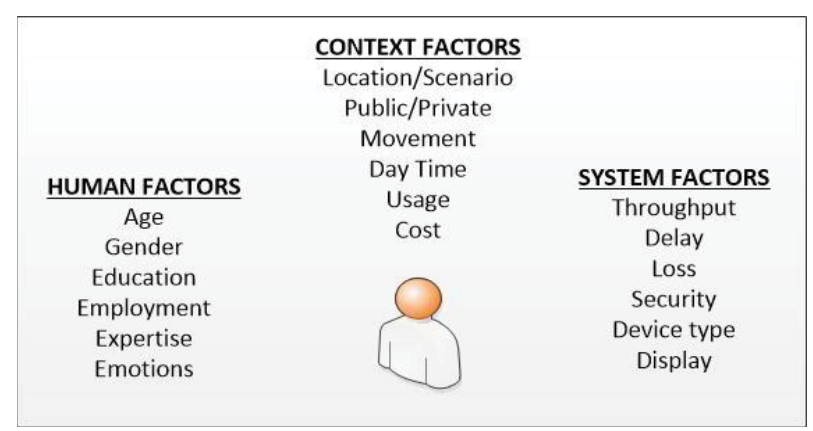

Figure 2. QoE dimensions and influence factors [9] 


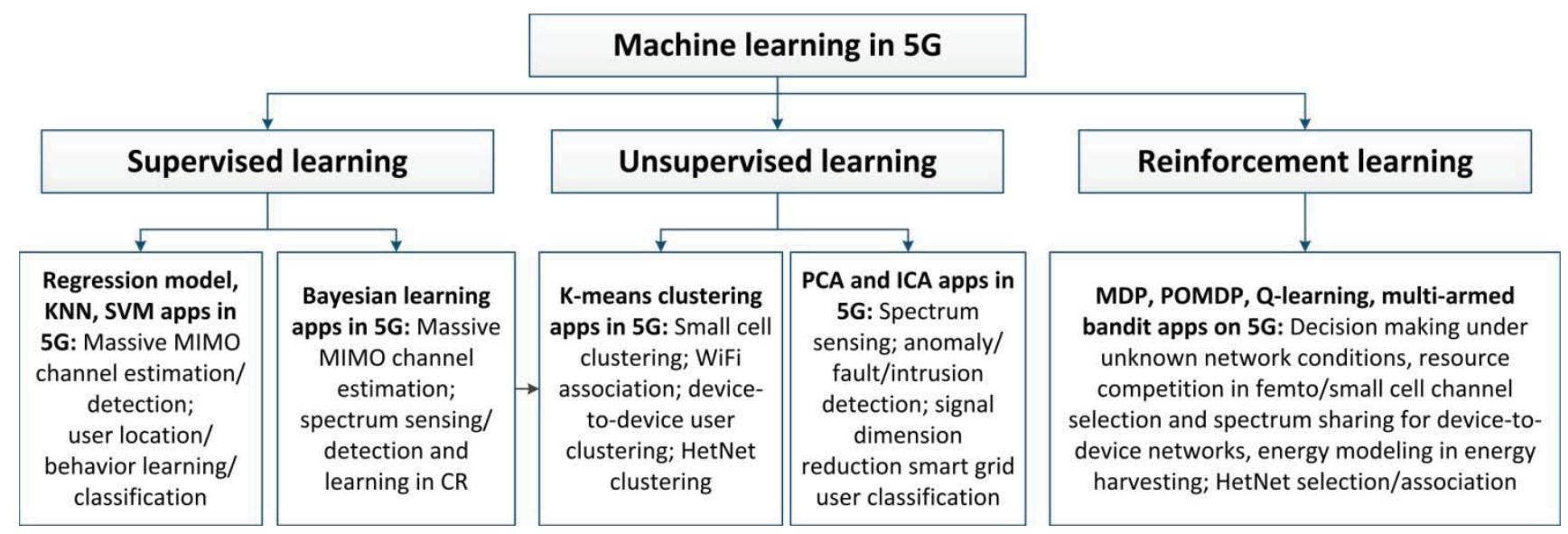

Figure 3. Machine-learning techniques and paradigms in 5G [11]

In this sense, a detailed description of the different $\mathrm{ML}$ algorithms and their application in the $5 \mathrm{G}$ environment is presented in [11] (Figure 3). The authors of this paper note the importance of selecting the appropriate learning type when using ML techniques since the goal of ML is to predict the output of an input turning observational data into a model that can be used for this prediction. Therefore, depending on the nature of the observational data, different types of learning can be distinguished:

- In supervised learning, each input value of the observational data is given with the corresponding output to form a training set. This set is used to learn a predictive function. Supervised learning is useful for classification and for regression problems.

- In unsupervised learning, only the input values are included in the observational data. The most common application of unsupervised learning is cluster analysis to find similarities between the input values and extract hidden patterns to group them into clusters.

- Reinforcement learning is based on dynamic iterative learning and decision-making processes. The learner is not told which actions to take but instead must determine those that yield the output closest to the target by successive trials.

\subsection{Standardization: Key to 5G}

Standardization bodies also envision the importance of defining new standards on QoS and ML for NGW ecosystems. In January 2018, a workshop on "Machine Learning for $5 G$ and beyond" was held in Geneva (Switzerland) in the context of the first meeting of the recently-launched ITU "Focus Group on Machine Learning for Future Networks including 5G" [5]. Three different working groups were established:

- WG1: Use cases, services \& requirements

- $W G 2$ : Data formats \& ML technologies

- WG3: ML-aware network architecture
In this context, ITU-T SG-13 has developed several Recommendations related to $5 \mathrm{G}$ QoS frameworks and $\mathrm{ML}$ techniques $[4,12]$ (Figure 4). In addition, ITU-T SG-12, the expert group responsible for the development of international standards for QoS and QoE, has been active in the editing and updating of related Recommendations to work around new mobile scenarios $[6,7]$. The 5G-PPP partnership, initiative between the European Commission and European ICT industry, is also working actively to define architectures, technologies and standards for NGW systems.

In spite of the significant advances in the definition of the $5 \mathrm{G}$ ecosystem and the identification of new QoE models and ML techniques for this environment, there is still a need for global QoS management models to be deployed in real-world NGW scenarios. In the following sections, an approach to solving this shortage is presented.

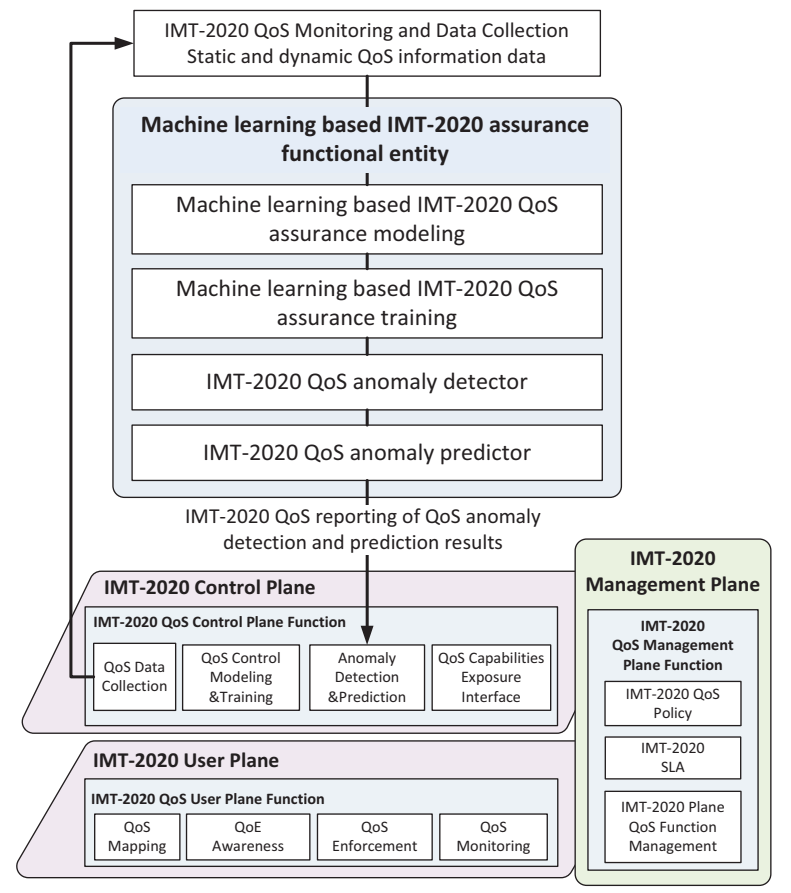

Figure 4. ITU model of ML-based QoS assurance [4, 12] 


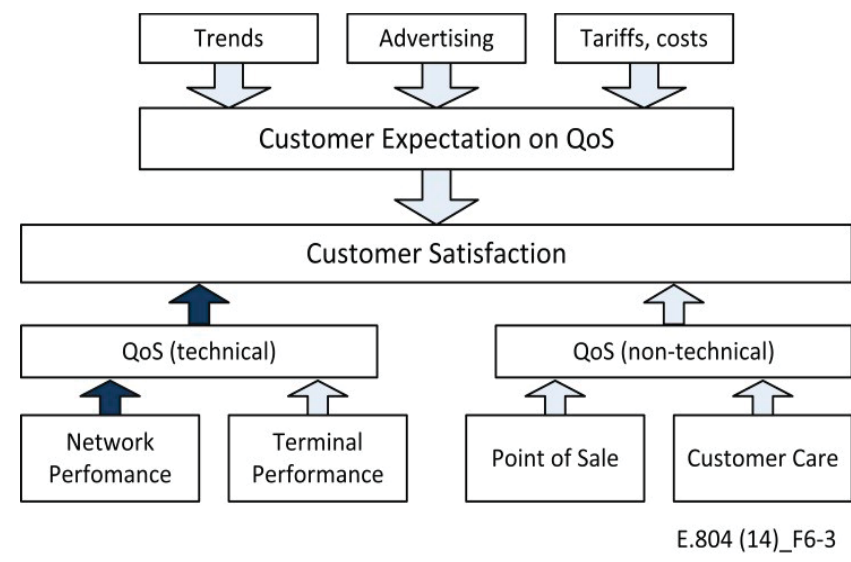

Figure 5. QoS relationships, from Recommendation ITU-T E.804 [6].

\section{QOS MANAGEMENT MODEL: QOXPHERE}

The QoS management model, QoXphere [13], has been adapted from its original architecture to include the new NGW ecosystem requirements. Nevertheless, the basic principles of the model remains; it still takes into account the four viewpoints of QoS of the ITU-T G.1000 QoS framework [8] and also the QoS aspects for mobile networks, as defined in ITU-T E.804 [6] are considered (Figure 5). Hence, the model is still organized in four different layers (Figure 6), though some of the QoS aspects considered in each layer have been updated (Figure 7) to fit with the new 5G QoS standardized framework.

The intrinsic QoS layer still identifies the KPIs to be used for the evaluation of objective QoS. Based on the specified class of service $(\mathrm{CoS})$, the key performance parameters (KPP) that contribute to each KPI must be identified to evaluate the NP. The results of the first layer analysis feed into the second layer of the QoXphere, where the QoS as perceived by the users (QoP) is estimated. This layer continues considering the ITU-T G.1000 four viewpoints of QoS. The identification of the KQIs of interest for the users is still the crucial challenge at this stage of the model.

The third layer of the model focuses on the evaluation of the assessed QoS. The user's satisfaction is modeled through the feed of the QoE provided by the second layer and the information about a user's expectations. The user's satisfaction values lead to identifying the key risk indicators (KRIs) to estimate the churn probability and establish the key business objectives (KBOs) that constitute the core of the upper layer of the model and analyses the QoBiz in terms of the profitability of the business models. This analysis may lead to "operational efficiency" actions, like defining new advertising procedures, new billing rates associated with new KQI/KPI objectives that will be reflected in the service level agreement (SLA). The new SLA will feed the user's expectations and requirements (through the required KQIs) that, at the same time, will aid the identification of the required KPIs to be considered and measured.

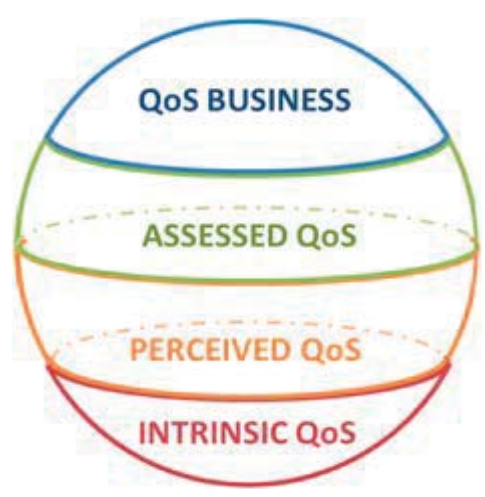

Figure 6. QoXphere management model

\section{MACHINE LEARNING METHODOLOGY}

The methodology we proposed is based on previous implementations of the QoXphere management model but has been enhanced for deployment in NGW ecosystems.

Due to the complex and heterogeneous nature of NGW scenarios, ML mechanisms are proposed to identify some of the QoS aspects and to formulate some of the relations between them. Gathering and collecting data to feed the learning algorithms is essential to infer rules to be applied both for the identification of all the key indicators and the specification of the relationships between the QoS aspects in the different layers of the model. In addition, the methodology will provide the procedures to continue feeding the model and find the gaps and "hot points" where intervention is required to improve the QoS and fulfill the QoE requirements. The ITU-T SG-13 QoS framework and ML-based QoS assurance, as proposed in [4, 12], have been taken into account when defining the proposed methodology, together with the ITU-T SG-12 principles of managing QoS, as defined in Recommendations ITU-T E.802[13] and G.1000 (Figure 8).
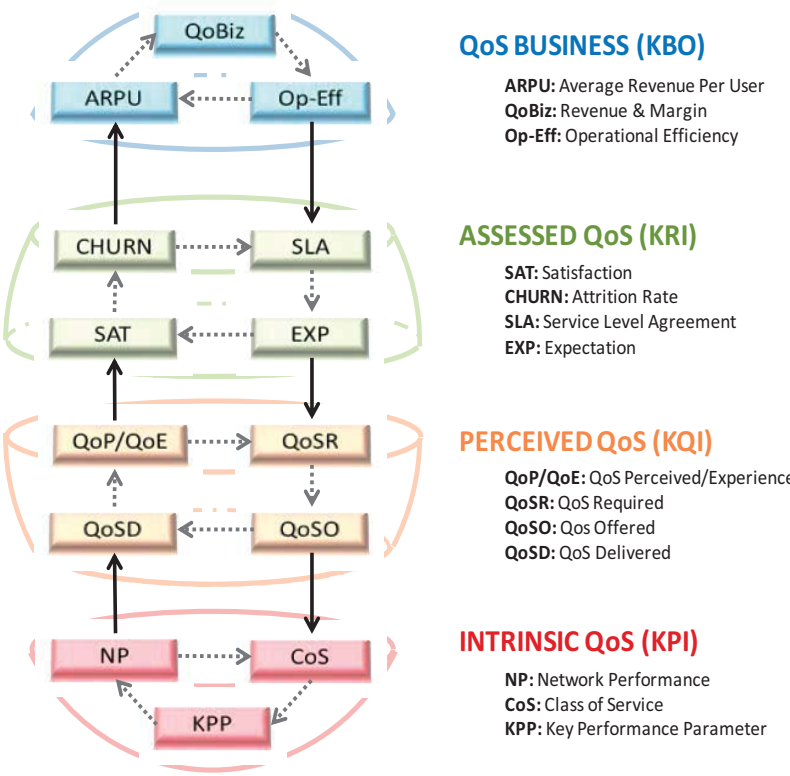

Figure 7. QoXphere: Layer structure 
As stated in the framework in Recommendation ITU-T E.802, for any QoS management model to be successful, it is crucial that the identification of the QoS criteria is relevant to the users (based on their requirements/expectations). In our previous work, we have considered the four models proposed in Recommendation ITU-T E.802 for the identification of the QoS criteria. In the various NGW scenarios, it is essential to determine the different QoE dimensions and influence factors that may aid in identifying the user's requirements. In the $5 \mathrm{G}$ wireless scenario, the network's response will be dependent on the users' behavior and many other contextual and noncontextual agents not controlled by the providers, which may have a considerable effect on the user's final satisfaction. For this reason, the first step proposed in the methodology is to understand the users' behavior in each of the scenarios to identify their requirements/expectations and determine the relevant QoS criteria. Therefore, since this first step is critical for the QoS management model to succeed, a combination of both contextual and noncontextual information is to be gathered through big data analysis. Unsupervised ML techniques (clustering) are proposed for inferring the different scenarios/profiles and for finding the user's context influence factors (the context extraction in Figure 8). In addition, inductive supervised learning is suggested to infer the rules to identify the QoS criteria and KQI relevant for the users. In this way, the complex procedure of surveying can be avoided except for the initial training period for the ML models to capture influence factors and user's requirements. Thus, the survey results will provide the particular cases of observation to draw the general rules predicted from a training set drawn from the context, system and human influence factors, together with the subjective information about the user's requirements and expectations gathered from surveys (i.e. the training set in Figure 8). Once the KPIs have been determined, the related KPPs will be specified for NP measurements based on the CoS and the ITU-T framework guidelines. At this stage, once the KPPs have been identified, the control of the "intrinsic QoS" may start (Control Plane in Figure 8). It is recommended to enable unsupervised ML techniques from the NP-gathered data to infer adequate radio/channel selection and detect faults and anomalies in the network behavior. This constitutes the first intervention point where corrective actions may be implemented to enhance the QoS (Anomaly Detection/Fault* in Figure 8).

For the study of the QoE (User Plane in Figure 8), the proposed method considers using also both the objective and subjective gathered data. Supervised machine-learning algorithms are suggested (regression model) for the correlation of the NP/QoE [10] using the survey's results and the NP collected data from probes, the correlation function between the QoE and NP can be deduced (see NP/QoE correlation model in Figure 8). The context and non-context influence factors will be also crucial when analyzing the user's QoE and for this reason, they feed again the training set to learn the rules that will provide the predicted QoE. The ML will avoid repeating the surveying complex process except for the time to capture the necessary observation data that will feed the training set process. This training set will be used to deduce the rules that will control the NP/QoE correlation model.

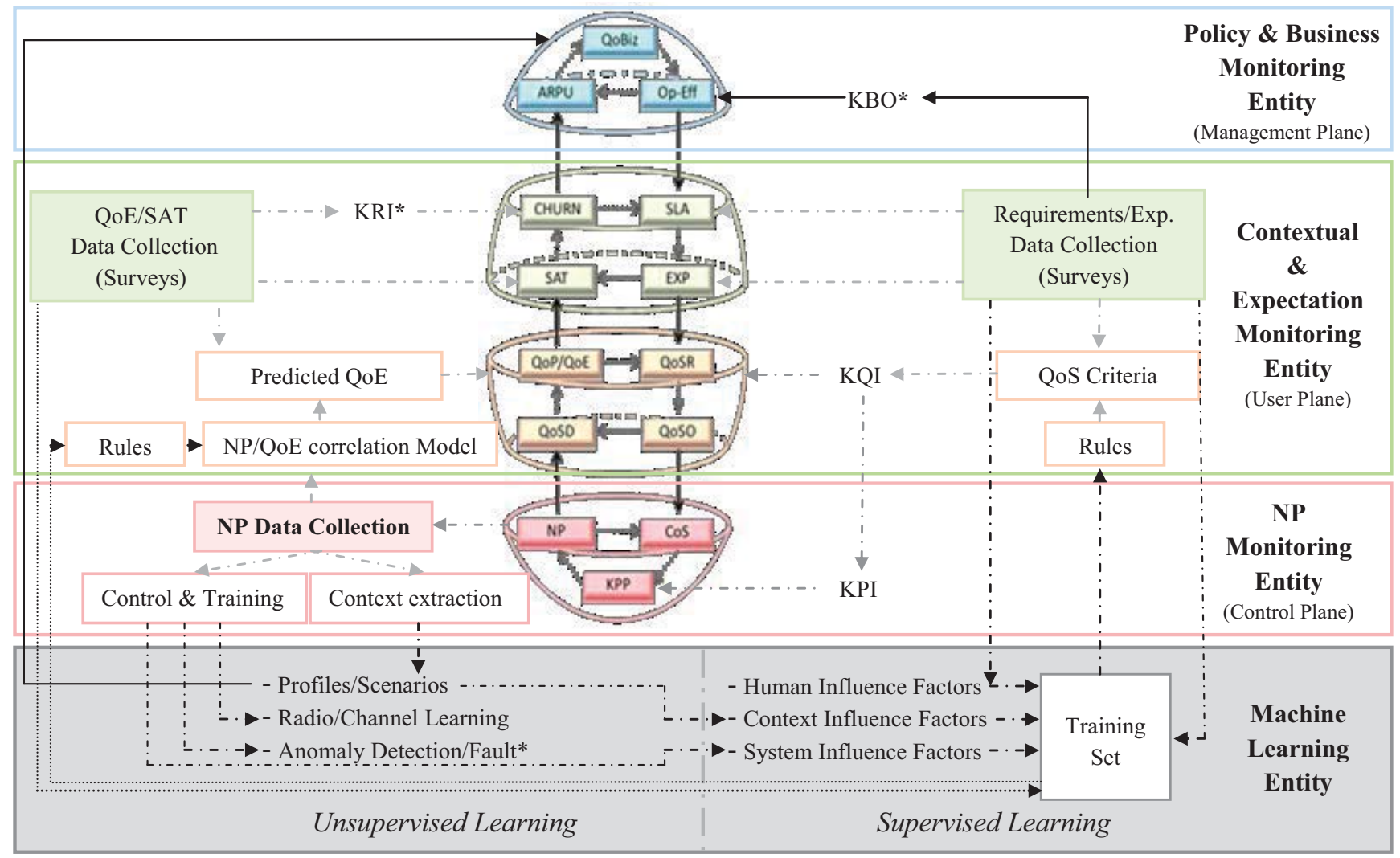

Figure 8. Proposed methodology for machine learning in 5G 
Based on the QoE results, the customer satisfaction model (CSAT) described in [14] will estimate the satisfaction of the user with the service. This constitutes the second "hot point" where corrective actions may be necessary based on the detected KRIs that may lead to churn and affect the business model, as shown in Figure 8.

Finally, the KBOs will be updated on a regular basis. As referred to in [15], the KBOs are derived from the business areas that are determined important for each company and they should be adjusted through the operational efficiency to increase revenue, reduce cost and improve customer experience. This constitutes the last intervention point where corrective actions may be required. Billing, advertising, fitting the QoS requirements and other measures should be analyzed to update the SLA, taking into account the outputs of the user plane and the control planes.

\section{CASE STUDY: IEEE 802.11 SCENARIOS}

In previous sections we have introduced the principles of the QoE enhancement methodology approach. In this section, we present the implementation of the proposal in a real next-generation wireless scenario: the Wi-Fi networks.

\subsection{Wi-Fi technology}

Wi-Fi technology, also known as IEEE 802.11, was originally designed to be a wireless local area network access technology which is to say intended only for small coverage areas. However, Wi-Fi has become a "ubiquitous access technology for mobile users" [1]. Consequently, it is now being considered as one of the key access technologies in the 5G HetNet ecosystem. Nevertheless, QoS management in this type of "unlicensed radio spectrum" wireless network may become very complex, especially when users' demands in terms of QoE are increasing.

IEEE 802.11e was developed to offer new QoS capabilities to the IEEE 802.11 WLAN networks. Under this amendment, new QoS mechanisms, such as the enhanced distributed channel access (EDCA) mechanism were introduced to Wi-Fi technology at the media access control (MAC) layer, enabling different classes of access categories (AC) in order to support the prioritization of distinct classes of services. Even though numerous research studies can be found for the implementation of this protocol in scientific literature, it is rarely used in real deployments. The reason is quite obvious: Wi-Fi QoS management is completely dependent on the behavior of users, the coexistence of networks and many other aspects that the provider cannot control. That is the reason why most Wi-Fi service providers currently resort to an "over-dimensioning" in the deployment of their access points (AP).

For the above, the Wi-Fi RAN has been selected to validate the proposed methodology to enhance QoE.

\subsection{Unsupervised ML: Wi-Fi patterns and scenarios}

For the identification of the different scenarios to be considered, DBSCAN clustering ML techniques were used. As a first stage, ML algorithms were applied only to the most relevant parameters from the extensive data set obtained from the probes (bandwidth consumed, transmission rate, number of data frames, frames in failure, etc). The results indicated distinct behavior patterns for, at least, three different types of scenarios:

- Commercial \& Business scenarios: shopping centers, restaurants, hotels, entertainment venues, etc.

- Public scenarios: schools, university campuses, cultural venues, museums, etc.

- Residential scenarios: both residential wireless access and shared agreement access.

Therefore, it was decided to carry out the study in three such real Wi-Fi scenarios:

- The CHQ building (https://chq.iel): to cover both business and commercial scenarios. This building, situated within the heart of Dublin city, holds different food and shopping spaces.

- Dublin Institute of Technology (http://www.dit.ie/): to cover public (e.g. educational campus) scenarios.

- The Gasworks Area: to cover residential scenarios.

Subjective and objective measurements were required to validate the methodology. Objective Wi-Fi information was collected through OptiWi-fi network probes described in [16]: NP data, location data, mobility patterns, number of access points (AP), number of clients, connection time, type of devices and other information, according to [17], was captured by the probes. Subjective information was gathered through user surveys: personal information (gender, age, occupancy, Internet expertise) together with the data related to the "Wi-Fi experience", both about the user's requirements/expectations and QoE/satisfaction with the service, were collected in the same survey. The dates of the test field in each of the scenarios are presented in Figure 9 where the surveying process was carried out in a oneweek period for each venue due to the complexity of the procedure and at least 50 participants took the survey in each of the scenarios. The network probes were deployed for a month in each of the venues to capture the required information about context and system influence factors.

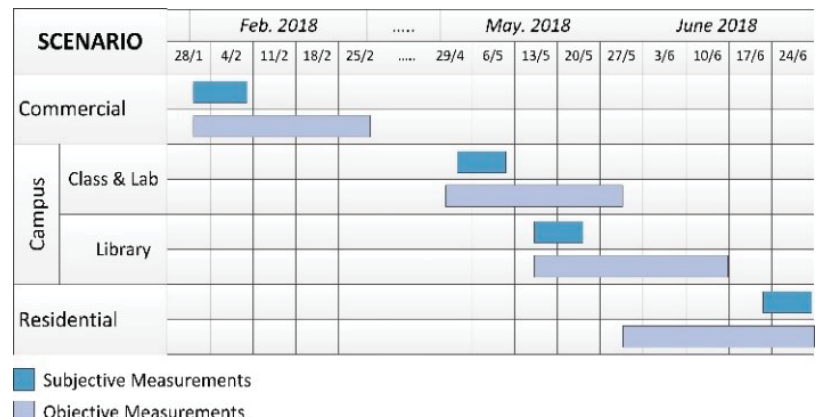

Figure 9. Test plan 


\subsection{QoS criteria and KQIs}

The data collected from the probes and the surveys shed light on the important differences between the users' influence factors in the different scenarios. In addition, the results of the surveys about the user's requirements for each of the scenarios also showed that the relevant QoS criteria and KQIs differ from one scenario to another (Figure 10). Although the use of ML to automatically update the KQIs has not been yet tested, it has been proven that it is essential given the number and changing nature of the influence factors.

\subsection{Control plane: Anomaly detector and predictor}

The data collected from the probes have been used, not only to learn about the users' behavior and extract the context information, but also to detect anomalies and enhance the channel selection process through unsupervised ML.

\subsection{User plane: Supervised ML}

Based on previous research studies [10], inductive supervised learning has been employed for the NP-QoE correlation model. The results of the surveys about user's QoE and satisfaction have fed the model to infer the rules to automatically predict the QoE based on NP and the influence factors. In future stages of the study ML techniques will be also implemented to enhance the satisfaction model (CSAT) described in [14].

\subsection{Results: Corrective actions to enhance QoE}

Though the validation of the methodology is still at a premature stage, the case study has revealed that the proposed methodology can be very useful to deploy the QoS management model and enhance the user's QoE. In fact, the results have indicated several corrective actions that could be implemented through ML in the scenarios under study:

- Commercial scenario: one of the most relevant KQIs in this scenario (streaming video/audio application performance) is affected for NP problems (continuous disruptions of the service). Corrective action: Analysis in NP data of AP capacity and use of ML to enhance the channel selection mechanism.

- Campus Scenario: Some client-association problems were found due to bad AP configuration. Furthermore, the students were dissatisfied with one of the KQIs (the ease of login) so the procedures around the login procedure should be revised. Corrective actions: Customize AP performance through ML techniques and revise login procedures according to the learned rules.

- Residential scenario: Lower cost and higher network speed were two of the key requirements in this scenario. Corrective actions: Customize residential Wi-Fi APs for optimal throughput through ML and enhance the business model using supervised ML through the survey's observation set.

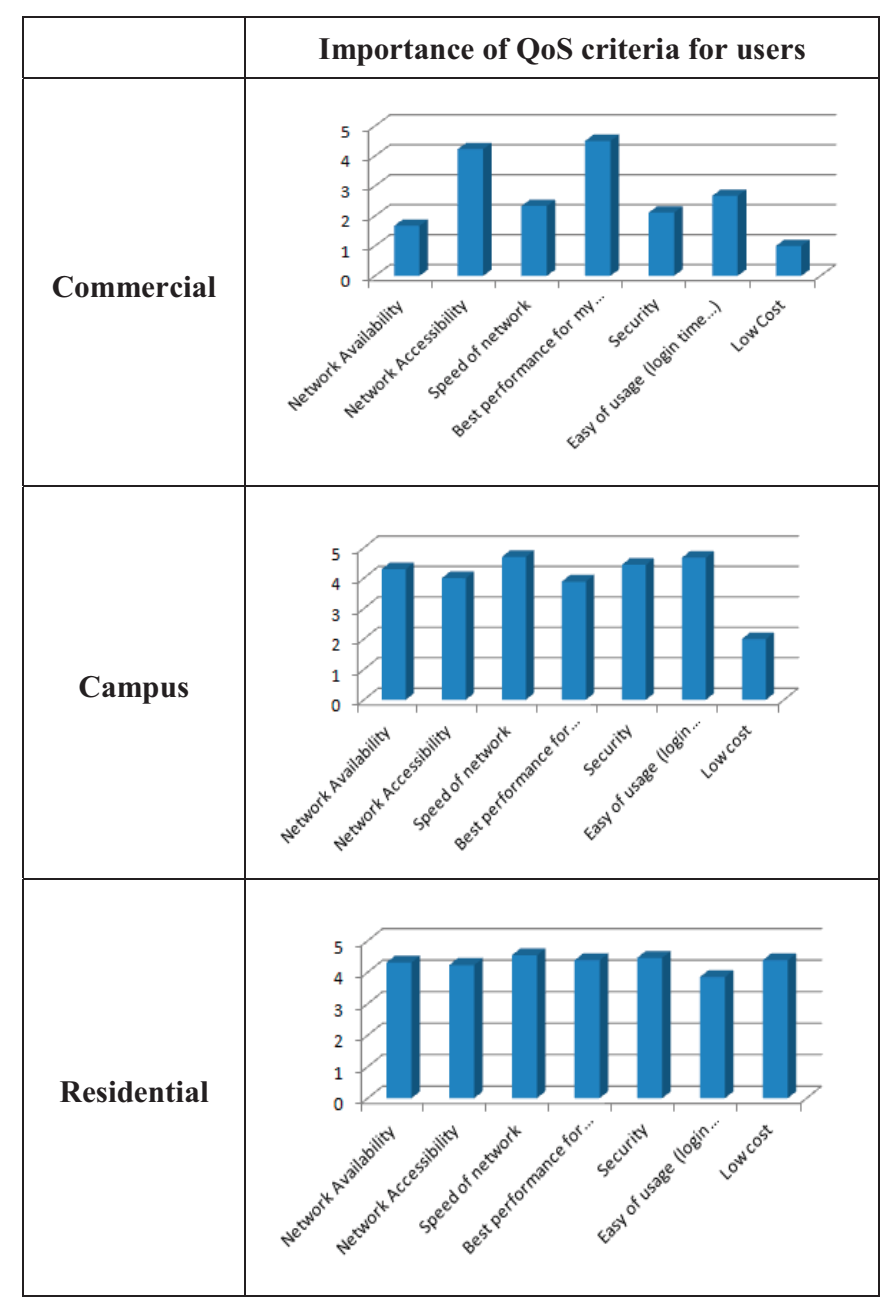

Figure 10. KQI relevance in different scenarios

\section{CONCLUSIONS}

In this paper a methodology to implement a global QoS management model for the next-generation wireless ecosystem, taking advantage of big data and ML techniques, has been presented.

Taking into account international standards, the QoEcentric approach makes use of supervised ML techniques in order to identify the KQIs relevant for the users. Unsupervised ML mechanisms are proposed for the identification of the user's influence factors, network performance anomalies, faults detection and channel selection enhancement. The approach links the NP and QoE via inductive $\mathrm{ML}$ techniques and provides the intervention points where corrective actions are required.

Although the definition of the methodology and the validation of the approach is still at an early stage, the results of the case study, carried out on a number of different Wi-Fi scenarios, reveal that the methodology may aid enhanced QoE in next-generation wireless environments. In addition, some recent studies suggest that ML techniques may be applied for customer retention to enhance the QoBiz and this will be also analyzed at future research stages of this proposal. 


\section{REFERENCES}

[1] L. Zhang, L. Zhao, Z. Wang, and J. Liu, "WiFi Networks in Metropolises: From Access Point and User Perspectives," IEEE Communications Magazine, vol. 55, pp. 42-48, 2017.

[2] K. Kaur, S. Kumar, and A. Baliyan, "5G: a new era of wireless communication," International Journal of Information Technology, pp. 1-6, 2018.

[3] Recommendation ITU-T, Y.3100: "Terms and definitions for IMT-2020 network," Geneva, 2017.

[4] Recommendation ITU-T Y.3170, "Requirements of machine learning based QoS assurance for IMT2020 networks," Geneva, 2018.

[5] ITU-T. (2017). Focus Group on Machine Learning for Future Networks including 5G. Available: https://www.itu.int/en/ITU-T/focusgroups/ml5g/

[6] Recommendation ITU-T, E.804: "Quality of service aspects for popular services in mobile networks," Geneva, 2014.

[7] ITU-T, "Draft new Recommendation ITU-T G.QoE$5 \mathrm{G}$ "QoE factors for new services in 5G network", 2018 .

[8] Recommendation ITU-T, G.1000: "Communications quality of service: A framework and definitions," Geneva, 2001.

[9] A. W. Yusuf-Asaju, Z. M. Dahalin, and A. Ta'a, "Framework for modelling mobile network quality of experience through big data analytics approach," Journal of Information and Communication Technology (JICT), vol. 17, pp. 79-113, 2018.
[10] S. Aroussi and A. Mellouk, "Survey on machine learning-based QoE-QoS correlation models," in International Conference on Computing, Management and Telecommunications (ComManTel), 2014, pp. 200-204.

[11] C. Jiang et al, "Machine Learning Paradigms for Next-Generation Wireless Networks," IEEE Wireless Communications, vol. 24, pp. 98-105, 2017.

[12] ITU-T, "Draft new Recommendation Y.IMT2020qos-fr: QoS support framework architecture for IMT-2020 networks", 2018.

[13] Recommendation ITU-T, E.802: "Framework and methodologies for the determination and application of QoS parameters," Geneva, 2007.

[14] E. Ibarrola, E. Saiz, L. Zabala, L. Cristobo, and J. Xiao, "A new global quality of service model: QoXphere," IEEE Communications Magazine, vol. 52, pp. 193-199, 2014.

[15] Recommendation ITU-T E.419: "Business oriented Key Performance Indicators for management of networks and services," Geneva, 2006.

[16] M. Davis, "A wireless traffic probe for radio resource management and QoS provisioning in IEEE 802.11 WLANs," in Proceedings of the 7th ACM international symposium on Modeling, analysis and simulation of wireless and mobile systems, Venice, Italy, 2004, pp. 234-243.

[17] M. Davis and T. Raimondi, "A novel framework for radio resource management in IEEE 802.11 wireless LANs," in Third International Symposium on Modeling and Optimization in Mobile, Ad Hoc, and Wireless Networks (WiOpt'05), 2005, pp. 139-147. 